\title{
Tribological Characteristics of Diamond-Like Carbon Based Double-Layers Film Prepared by DC-Plasma Ionization Deposition
}

\author{
Tsuyoshi Mano, Shogo Tanaka, Makoto Takagi, Osamu Sugiyama \\ and Osamu Takai* \\ Shizuoka Industrial Research Institute, Fuji Technical Support Center, \\ Shizuoka Prefectural Government, 2590-1 Ohbuchi, Fuji, Shizuoka 417-8550, Japan \\ * EcoTopia Science Institute, Nagoya University, Furo-cho, Chikusa-ku, Nagoya \\ 464-8603, Japan
}

Keywords : Diamond-like carbon, Friction, Tribology, Raman scattering spectroscopy

\section{Introduction}

It is well-known that diamond-like carbon (DLC) films have excellent properties, such as high hardness, high corrosion durability and wear resistance [1-3]. However, the bonding-strength of DLC films with some kinds of substrates is weak, which will spoil their good characteristics especially in high load applications. Lack of durability, in many cases, originates from high internal stress [4-6]. Some researchers successfully improved the durability through the reduction of the internal stress [7-9], although, a decrease of hardness was accompanied with it due to the coexistence of ductile metals.

We have prepared original DLC based double-layers film with good adhesion with substrates and high hardness by the DC-plasma ionization deposition method [10]. The film consists of a DLC upper layer and a compositionally graded lower layer of silicon and carbon compounds with decreasing $\mathrm{C} / \mathrm{Si}$ atomic ratio to the substrate. Furthermore, the depositing process of the double-layers film can be performed sequentially without the extinction of plasma. In this study, we focused on the tribological properties of the DLC based double-layers film and clarified the mechanism of the excellent anti-friction performance.

\section{Experimental}

Two types of DLC films were formed: one was of single-layer structure (monolithic DLC film; abbreviated as sl-DLC), and the other was of double-layers structure (a DLC layer on a graded composition layer; abbreviated as DLC/GCL). In the sl-DLC, the DLC layer was formed directly on the substrate. In the DLC/GCL, the GCL was formed onto the substrate first and then the DLC layer was done on it.

Carbon tool steel plates, AISI W1-8, were used as substrates. Ar ion bombardment for $20 \mathrm{~min}$ was performed in the vacuum chamber just prior to the deposition process.

Film deposition operation was carried out using a DC-plasma ionization deposition apparatus [11,12]. The schematic diagram of the process chamber of the apparatus is shown in Fig. 1.

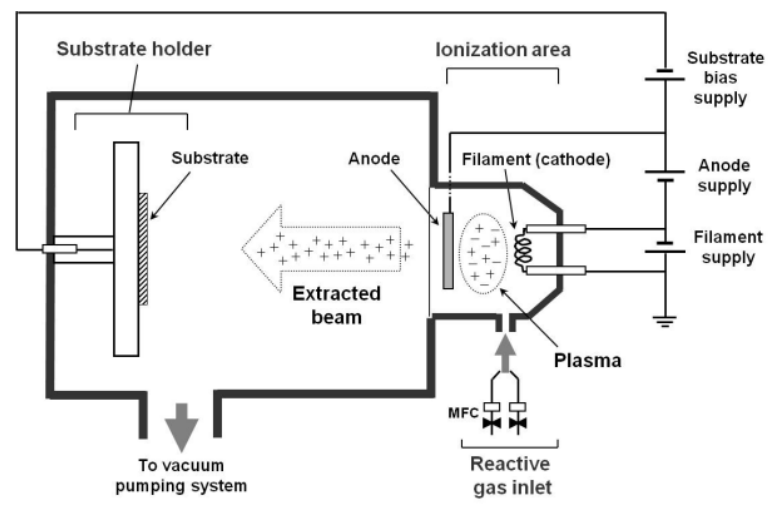

Fig. 1. Film deposition process using the DC-Plasma ionization deposition. 
The chamber is equipped with an ionization area, a reactive-gas inlet and a substrate holder. The anode and hot filament (cathode) in the ionization area generate a dense plasma. Source gasses of the DLC layer and the graded composition layer (GCL) were benzene $\left(\mathrm{C}_{6} \mathrm{H}_{6}\right.$, $>99.9 \%$ in purity) vapor and the vapor mixture of benzene and hexamethyldisiloxane (abbreviated as HMDSO; $\left[\left(\mathrm{CH}_{3}\right)_{3} \mathrm{Si}\right]_{2} \mathrm{O},>99.0 \%$ in purity), respectively. In the preparation of the GCL, an adding volume ratio of HMDSO to benzene was decreased in a stepped mode. Further details of the deposition system and the structure of films can be found elsewhere [10,11]. Total thicknesses of the sl-DLC and the DLC/GCL were fixed at about $400 \mathrm{~nm}$, by controlling deposition period. In the DLC/GCL, the thickness of GCL was about $70 \mathrm{~nm}$.

Friction and wear properties were measured with a ball-on-disk type wear tester in dry air at room temperature. A carbon-chrome steel ball (AISI 52100, $3.0 \mathrm{~mm}$ in diameter), was used as a counterpart ball. The substrate with the film on it was set in the tester and rotated under a constant normal load of $30 \mathrm{~N}$ by the ball, which created an initial Hertzian mean pressure of approximately $2.0 \mathrm{GPa}$. A sliding velocity was $80 \mathrm{~mm} / \mathrm{s}$, and numbers of sliding cycles were 100000 and 300000 (wear distances; $942.5 \mathrm{~m}$ and $2828 \mathrm{~m}$, respectively). The coefficient of friction was measured during the tests. The wear tracks and scars were characterized with a scanning electron microscope (SEM) and an electron probe microanalyzer (EPMA). A Raman scattering spectroscope operated with an Ar laser having a wavelength of $514.5 \mathrm{~nm}$ was also used for the clarification of wear mechanism. The analyzing area was approximately $10 \mu \mathrm{m}$ in diameter.

\section{Results and discussion}

Figure 2 shows the variation of coefficient of friction for the sl-DLC and DLC/GCL with increasing sliding cycles in the ball-on-disk test. The result of the AISI W1-8 steel substrate is also provided in this figure as a reference. The substrate showed high coefficient of friction of about 0.8 from the initial stage of the test. The coefficient of sl-DLC was relatively low, about 0.2 , from 0 to 60000 cycles, however, it increased abruptly at 60000 cycles towards to 0.8 , suggesting that the DLC film was almost lost and the ball contacted with the substrate directly. The SEM observation of the wear track

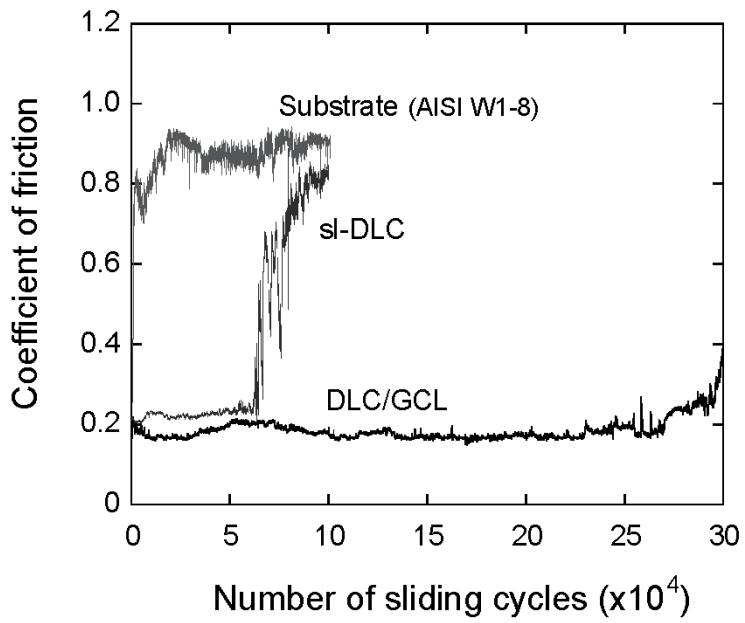

Fig. 2. Variation in the coefficients of friction of the sl-DLC, DLC/GCL and the AISI W1-8 steel substrate with increasing number of sliding cycles in the ball-on-disk test.

confirmed the peeling-off of the sl-DLC film at about 60000 cycles. On the other hand, the coefficient of the DLC/GCL maintained low value, about 0.2 , up to 250000 cycles, showing a long-term durability under heavy loaded friction environment.

In order to clarify the good tribological property of the DLC/GCL film, wear tracks on the film and wear scars on the counterpart ball after long-duration test of 300000 cycles were analyzed. Some transformed regions, such as one denoted as A in Fig. 3 (a), were observed around the edge of wear scars on the ball. Since the major element existed in the A region was Carbon from EPMA, it was considered to be a transferred DLC layer. Further, fiber-like matters, such as B in Fig. 3 (b), were observed, which also consisted of $\mathrm{C}$, suggesting they were the remnants of the DLC/GCL film. These carbon matters were scarcely observed after 100000 cycles, and also not found for the sl-DLC after tested at 100000 cycles. As shown in Fig. 4 , the Raman spectra of the transferred layer $A$ and the film remnant $B$ differed from that of the as-prepared DLC/GCL film, which was assigned to typical monolithic hydrogenated amorphous carbon [11]. Wave numbers of D-peak (around $1550 \mathrm{~cm}^{-1}$ ) and G-peak (around $1350 \mathrm{~cm}^{-1}$ ) of B shifts to higher, and height of D-peak is higher than the DLC/GCL. It is guessed that high amount of $s p^{2}$-bonding existed in the film remnants on the wear scar. Furthermore, the 

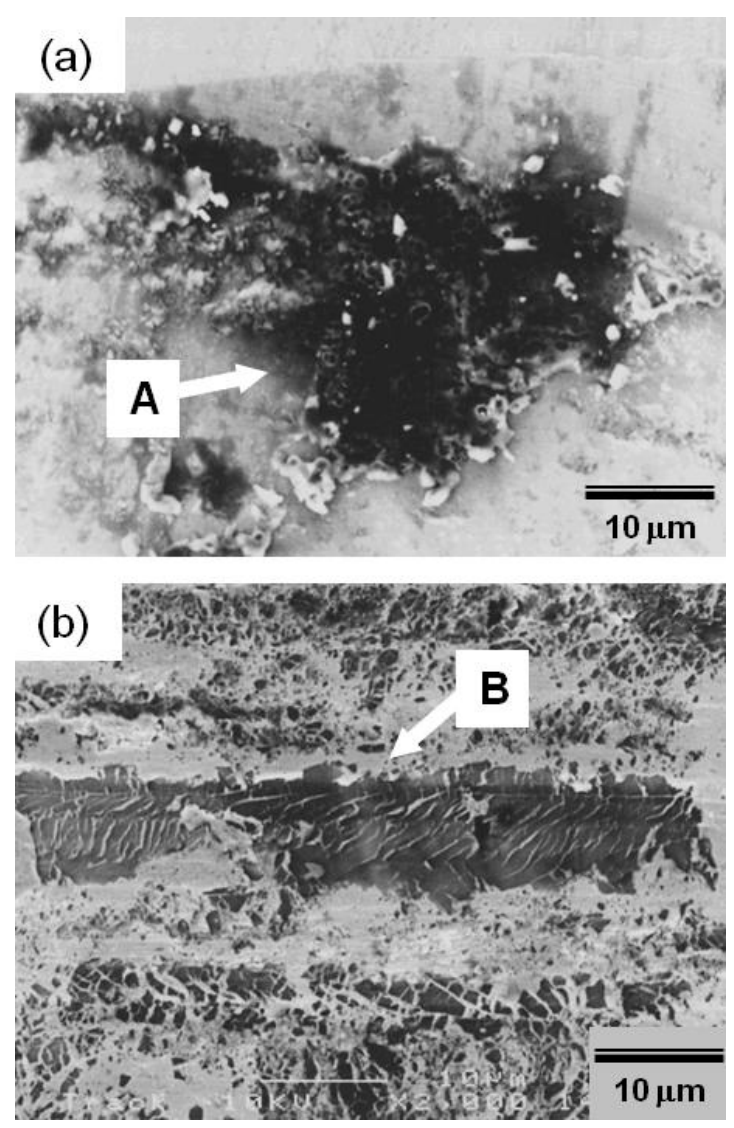

Fig. 3. SEM images of contacting regions after 300000 cycles in the long-duration ball-on-disk test: (a) is wear scars on counterpart ball; (b) is wear track on the DLC/GCL.

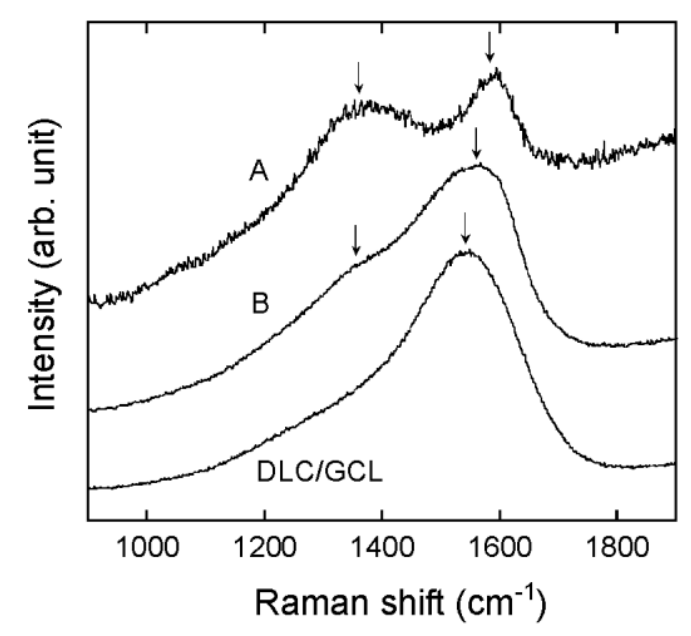

Fig. 4. Raman spectra of the transfer layer at the position $A$ in FIG. 3 (a) and the film remnant at B in FIG. 3 (b). A spectrum of as-prepared DLC/GCL film is also provided as a reference. shape of A-spectra with D- and G-peaks at around $1585 \mathrm{~cm}^{-1}$ and $1360 \mathrm{~cm}^{-1}$ is similar to a typical graphite structure being fully made of $s p^{2}$-bonding. It is known that amount of $s p^{2}$-bonding in a DLC film increases through heat treatment [13]. The friction energy generated through the severe wear test must have promoted the formation of $s p^{2}$-bonding or the graphitic structure with high lubricity, which have played an important role in the enhancement of the anti-friction property.

A. Erdemir et al. researched on the formation mechanism and properties of a transferred layer on counterpart balls [14]. They reported that the transferred layer containing graphitic structure, which had formed in a long-duration ball-on-disk test under the load of $5 \mathrm{~N}$ (Hertzian mean pressure was about $0.54 \mathrm{GPa}$ ), had contributed the suppression of increase in the coefficient of friction,. Also for the DLC/GCL in our research, no steep increase of the coefficient was observed in the long-duration test under the severe conditions at the load of $30 \mathrm{~N}$ (Hertzian mean pressure was about $2.0 \mathrm{GPa}$ ) up to 250000 cycles. Since similar graphitic structure was confirmed in both the film remnant and the transferred layer, the conservation of the low-level coefficient must be due to them. Slow increase of the coefficient after 250000 cycles can be regarded as the gradual peel-off of the film remnants. This is much contrast to the abrupt rising of the coefficient for the sl-DLC, which seemed to be caused by the sudden peel-off of the film owing to its high internal stress. The graded composition layer in the DLC/GCL must have reduced the internal stress to make the film remnants stably bonded to the substrate and not to cause the catastrophic peel-off. In other words, existence of the graded composition layer brought the high adhesion strength between the DLC/GCL and the substrate to give a long duration time and heat energy, which further served the concentration of graphitic $s p^{2}$-bonding to give high lubricity.

\section{Conclusion}

A DLC based double-layers film was produced by the DC-plasma ionization deposition method and their tribological characteristics on carbon steel were studied. The double-layers film, which was much effective to reduce its internal stress, showed excellent wear resistance compared with a single-layer DLC film: it maintained low coefficient of friction almost throughout a ball-on-disk test (load of $30 \mathrm{~N}$, 
sliding number of 300000 cycles). Low internal stress resulted in the high adhesion strength between the double-layers film and the substrate, which offered a sufficient time for the formation of graphitic $s p^{2}$-bonding by obtaining friction energy during the severe wear test. The graphitic structure was further transferred to the counter ball, and played a role as a lubricant to enhance the wear resistance of the film. It was concluded that the structural gradation of the double-layers film brought the excellent tribological characteristics.

\section{References}

1. D. R. McKenzie, Rep. Prog. Phys., 59 (1996) 1611.

2. J. Robertson, Prog. Solid. Chem., 21 (1991) 199.

3. H. Liu, A. Tanaka, K. Umeda, Thin Solid Films, 346 (1999) 162.

4. J. Robertson, Diamond Relat. Mater., 2 (1993) 984.

5. K. J. Clay, S. P. Speakman, N. A. Morrison,
N. Tomozeiu, W. I. Milne, A. Kapoor, Diamond. Relat. Mater. , 7 (1998) 1100.

6. R. G. Lacerda, F. C. Marques, Appl. Phys. Lett., 73 (1998) 617.

7. D. F. Franceschini, C. A. Achete, F. L. Freire Jr, Appl. Phys. Lett., 60 (1992) 3229.

8. H. Fukui, H. Ohara: Surf. Coat. Technol. , 146-147 (2001) 378 .

9. M. M. Morshed, D. C. Cameron, B. P. McNamara,M. S. J. Hashimi, Surf. Coat. Technol., 169-170 (2003) 254.

10. T. Mano, O. Sugiyama, Y. Shibuya, H. Nakayama, O. Takai, Mater. Trans., 45 (2004) 1601.

11. T. Mano, O. Sugiyama, Y. Shibuya, O. Takai, J. Ceram. Soc. Japan, 112-1 (2004) 1517.

12. C. Weissmantel, K. Bewilogua, K. Breuer, D. Dietrich, U. Ebersbach, H. J. Erler, B. Rau, G. Reisse, Thin Solid Films, 96 (1982) 31.

13. M. N. Gardos, B. L. Soriano, J. Mater. Res., 5 (1990) 2599.

14. A. Erdemir, C. Bindal, J. Pagen, P. Wilbur, Surf. Coat. Technol., 76-77 (1995) 559. 